Programa de Pós-Graduação em Engenharia de Produção - PPGEP

Laboratório de Qualidade de Vida - LaQVida

Universidade Tecnológica Federal do Paraná - UTFPR

Ponta Grossa - PR - Brasil

v. 05 , n. 04 , out./dez. 2013 , p. $44-54$

DOI: $10.3895 / \mathrm{S} 2175-08582013000400006$

\section{REVISTA BRASILEIRA DE QUALIDADE DE VIDA}

\title{
Estilo de vida e indicadores organizacionais e de produtividade em uma empresa industrial da Região Nordeste do Brasil
}

\section{Lifestyle and productivity and organizational indicators in an industrial company in the Northeast of Brazil}

\author{
Regimênia Maria Braga de Carvalho \\ Serviço Social da Indústria - SESI - Campina Grande - Brasil \\ regimeniacarvalho@fiepb.org.br \\ Alberto José Niituma Ogata \\ Centro Universitário São Camilo - CUSC - São Paulo - Brasil \\ albertoogata@gmail.com \\ Kleber Barbosa Silva \\ Serviço Social da Indústria - SESI - Campina Grande - Brasil \\ kleberbarbosa@fiepb.org.br \\ Bruno Teixeira Barbosa \\ Bolsista de Desenvolvimento Tecnológico Industrial do CNPq - Nível C - Brasil \\ btbarbosaa@gmail.com
}

\section{RESUMO}

OBJETIVO: O presente estudo tem por objetivo apurar a relação entre os indicadores de produtividade, com especial ênfase para o presenteísmo e fatores de risco em saúde e estilo de vida. Adicionalmente, será elaborado um instrumento de avaliação de impacto de fatores de risco em saúde e estilo de vida sobre a produtividade de trabalhadores da indústria.

MÉTODOS: O estudo consistiu de uma amostra de trabalhadores $(n=423)$ de uma grande empresa têxtil da Região Nordeste do Brasil. A este grupo foi aplicado um questionário baseado em questões de gênero, escolaridade, fatores de risco para a saúde e estilo de vida. Em relação à prática de atividades físicas foi utilizado o instrumento International Physical Activity Questionnaire (IPAQ) curto. Os fatores de risco em saúde são referidos pelos trabalhadores, sendo utilizados os parâmetros propostos pela pesquisa Vigilância de Fatores de Risco e Proteção para Doenças Crônicas por Inquérito Telefônico (VIGITEL) (MINISTÉRIO DA SAÚDE, 2009), conduzido pelo Ministério da Saúde. A carga de trabalho foi apurada através do questionário adaptado de Qualidade de Vida Profissional (QVP), desenvolvido por Cabezas-Peña (1999).

RESULTADOS: Aproximadamente 63\% dos funcionários apresentam três ou mais fatores de risco. Há uma forte relação entre o número de fatores de risco e inatividade física. Os funcionários que relataram presenteísmo são 6,5 vezes mais propensos a se ausentar do trabalho. Há uma relação estatística significativa entre os trabalhadores do sexo feminino com nível elevado de estresse e presenteísmo.

CONCLUSÕES: A promoção do estilo de vida saudável, com especial ênfase para a atividade física constitui-se numa ferramenta poderosa para a melhoria dos indicadores analisados. O presente 
estudo revelou que as pessoas com maior idade e com mais fatores de risco tem risco aumentado pela inatividade física.

PALAVRAS-ChAVE: Estilo de vida. Saúde. Atividade Física. Produtividade.

\begin{abstract}
OBJECTIVE: The aim of this study is to investigate the relationship between indicators of productivity, with special emphasis on presenteeism, and risk factors in health and lifestyle. Additionally, an instrument will be developed for assessing the impact of risk factors on health and lifestyle on the productivity of industrial workers.
\end{abstract}

METHODS: The study consisted of a sample of workers $(n=423)$ of a large textile company in Northeastern Brazil. This group was given a questionnaire based on gender, education, life risk factors and lifestyle. Regarding physical activity, Physical Activity Questionnaire (IPAQ) was used. The risk factors are referred by health workers, based on the parameters proposed by a survey named Vigilância de Fatores de Risco e Proteção para Doenças Crônicas por Inquérito Telefônico (VIGITEL) $^{1}$ (MINISTÉRIO DA SAÚDE, 2009), conducted by the Ministry of Health. The workload was determined by a questionnaire adapted from Qualidade de Vida Profissional (QVP) ${ }^{2}$ developed by Cabezas-Peña (1999).

RESULTS: Approximately $63 \%$ of employees have three or more risk factors. There is a strong relationship between the number of risk factors and the physical inactivity. Employees who reported presenteeism are 6.5 times more likely to be absent from work. There is a statistically significant relationship between female workers and high level of stress and presenteeism.

CONCLUSIONS: The promotion of healthy lifestyle, with a focus on physical activity is a powerful tool for the improvement of the analyzed indicators. The present study revealed that people with older age and with more risk factors are at increased risk for physical inactivity.

KEYWORDS: Lifestyle. Health. Physical Activity. Productivity.

\title{
1. Introdução
}

Atualmente, no Brasil, as doenças crônicas não transmissíveis (infarto do miocárdio, derrames cerebrais, diabete tipo II e câncer) são as principais causas de morte em adultos. De acordo com a Organização Mundial da Saúde (OMS, 2010), aproximadamente $80 \%$ dos casos de doenças cardiovasculares e diabetes e $40 \%$ dos casos de câncer são evitáveis através da mudança do estilo de vida, tomando como base a criação de hábitos que incluam a prática de atividade física, alimentação saudável, eliminação do hábito de fumar e a adoção de comportamentos preventivos (como realizar exames preventivos e não dirigir quando ingerir bebidas alcoólicas).

O Ministério da Saúde está realizando, desde 2006, o estudo Vigilância de Fatores de Risco e Proteção para Doenças Crônicas por Inquérito Telefônico (VIGITEL) (MINISTÉRIO DA SAÚDE, 2009), para monitorar vários indicadores nas capitais dos Estados e do Distrito Federal. Os números de 2009, publicados em 2010, revelaram que, em dados aproximados, somente 30\% dos brasileiros ingerem cinco porções por semana de frutas, vegetais e legumes e $33 \%$ comem carne vermelha com gordura visível. Por outro lado, somente $32 \%$ dos brasileiros relataram praticar atividade física no tempo livre e no deslocamento e apenas $14 \%$ no tempo livre. A consequência é que $46 \%$ dos adultos entrevistados estão com excesso de peso e 13,9\% são obesos. A constatação de que há uma tendência no aumento do número de obesos no Brasil tem preocupado os pesquisadores e os profissionais da saúde.

\footnotetext{
${ }^{1}$ Surveillance of risk and protective factors for chronic diseases through telephone survey.

${ }^{2}$ Professional Life Quality.
} 
De acordo com a OMS (2010), a saúde dos trabalhadores, bem como a sua segurança e o seu bem-estar são motivos de preocupação de milhares de profissionais no mundo todo.

Bergstrom et al. (2009) e Burton et al. (2006) analisam o absenteísmo (faltas ao trabalho), o presenteísmo (estar presente no trabalho, mas não ser produtivo), com os custos com assistência médica, acidentes no trabalho e aposentadorias precoces por problemas ocupacionais e mostram que as intervenções no ambiente de trabalho são eficazes e melhoram todos esses indicadores. Os fatores de risco para doenças crônicas, como obesidade, sedentarismo, tabagismo, maus hábitos alimentares e estresse têm uma relação direta com a produtividade (GOETZEL et al., 2009).

A gestão de saúde e bem-estar em uma empresa busca atingir níveis ideais de saúde dos trabalhadores com consequentes resultados positivos de produtividade. Os programas devem seguir estratégias e táticas que justifiquem os investimentos (GOETZEL et al., 2009).

A questão do presenteísmo é um fator importante associado à produtividade e que deve merecer a atenção dos gestores e planejadores em saúde. De acordo com Schultz, Chen e Edington (2009), a preocupação no que tange os problemas de saúde do trabalhador que não culminam no absenteísmo está ligada à redução de produtividade presente antes e depois da ausência no trabalho, ou simplesmente 'ir ao trabalho apesar de se sentir doente'.

Por preceder o absenteísmo, o presenteísmo demanda uma maior atenção por parte dos gestores, isto é, se o trabalhador não for apoiado para se sentir melhor ou lidar com algum fator relacionado à saúde, o quadro pode se agravar e ele começar a faltar ao trabalho. Este quadro pode levar a erros no cumprimento das tarefas, redução na produtividade e comprometimento na qualidade dos produtos e serviços.

Deve-se lembrar de que não há somente fatores pessoais que levam ao presenteísmo. Há componentes organizacionais associados, como o excesso de demandas no trabalho, pressão de tempo, estresse, insegurança no emprego ou dificuldade de reposição (o trabalho tem que ser feito após o retorno às atividades). Além disso, há questões pessoais que não estão relacionadas à saúde que podem estar ligadas ao presenteísmo, como as dificuldades financeiras, a falta de limites pessoais relacionados ao equilíbrio da vida pessoal e profissional, engajamento excessivo com o trabalho e a faixa etária (BERGSTROM et al., 2009).

Neste contexto, os programas de qualidade de vida no trabalho têm grande importância na abordagem das questões relacionadas à produtividade do fator humano, notadamente no que se refere ao presenteísmo.

Recente pesquisa de âmbito nacional analisou o estilo de vida e hábitos de lazer dos trabalhadores das indústrias brasileiras. Este levantamento, realizado pelo Serviço Social da Indústria (SESI), envolveu 2.775 empresas e 47.886 trabalhadores. Observou prevalência de consumo elevado do álcool $(33,0 \%)$, de falta de atividade física no lazer $(45,4 \%)$, excesso de peso corporal $(40,5 \%)$ e falta de consumo adequado de frutas e hortaliças $(60,0 \%)$. Estes resultados corroboram que abordar questões relacionadas ao estilo de vida é estratégico para o setor industrial, para manter a produtividade e a saúde dos trabalhadores e preservar a competitividade no cenário internacional (NAHAS et al., 2008).

O presente estudo tem por objetivo apurar a relação entre os indicadores de produtividade, com especial ênfase para o presenteísmo e fatores de risco em saúde e estilo de vida; adicionalmente será elaborado um instrumento de avaliação de impacto de fatores de risco em saúde e estilo de vida sobre a produtividade de trabalhadores da indústria.

\section{Métodos}

A pesquisa foi realizada em uma indústria do setor têxtil no Estado da Paraíba, com 1628 funcionários; deste total, 423 trabalhadores foram selecionados como amostra do estudo. Foram incluídos aqueles que estavam em horário de serviço, excluindo-se os que não tivessem um ano completo na empresa, como também os trabalhadores com deficiência auditiva. No decorrer do estudo, algumas limitações (mudança de setor dos trabalhadores, turnover, férias, afastamento e 
desistência na participação) impossibilitaram a utilização da amostra na medição de determinadas variáveis.

A coleta de dados, realizada no período de março/abril de 2010, respeitou o horário de funcionamento da empresa e aconteceu nos três turnos de trabalho: manhã, tarde e noite. A participação da amostra na pesquisa esteve condicionada à disponibilidade dos trabalhadores ao longo das jornadas de trabalho e, portanto, o grupo selecionado não revela necessariamente a estratificação dos diferentes setores da empresa; ressalte-se que o estudo não visa analisar o perfil da companhia, mas o do grupo pesquisado.

O instrumento utilizado no estudo foi um questionário que contempla questões referentes a dados sociodemográficos (idade e gênero), estilo de vida (atividade física no lazer, ingestão de frutas, vegetais e hortaliças, carne vermelha com gordura visível, tabagismo e estresse), fatores de risco em saúde - morbidade referida (hipertensão arterial, diabetes, hipercolesterolemia) e variáveis organizacionais e de produtividade (carga de trabalho, motivação intrínseca, presenteísmo, absenteísmo).

Considerou-se como alto risco, relacionado à má nutrição, os indivíduos com baixo consumo de frutas, verduras e hortaliças (menos que duas vezes por semana) e consumo de carnes vermelhas (mais de três vezes por semana), inclusive com gordura visível, de acordo com critério adaptado de Goetzel et al. (2009).

Em relação à prática de atividades físicas foi utilizado o instrumento International Physical Activity Questionnaire (IPAQ) curto, validado no Brasil por Matsudo et al. (2001). Foi considerado sedentário o indivíduo que não realizou nenhuma atividade física por pelo menos dez minutos durante a semana; insuficientemente ativo o indivíduo que realiza atividade física por pelo menos dez minutos por semana, porém insuficiente para ser classificado como ativo. Foram separados dois grupos: sedentário e insuficientemente ativo (<150 min./sem.) e ativo (> $150 \mathrm{~min} . / \mathrm{sem}$.). Foi considerado ativo o indivíduo que cumpriu as recomendações vigorosas (3 dias/sem e 20 min./sessão), moderada ou caminhada (5 dias/sem e $30 \mathrm{~min} . / \mathrm{sessão)} \mathrm{ou} \mathrm{qualquer} \mathrm{atividade} \mathrm{somada}$ (5 dias/sem e $150 \mathrm{~min} . / \mathrm{sem}$ ).

A carga de trabalho foi apurada através do questionário adaptado de Qualidade de Vida Profissional (QVP-35) desenvolvido por Cabezas-Peña (1999) e validado por Guimarães et al. (2004).

O presenteísmo foi apurado em conformidade com o proposto e utilizado por Bergstrom et al. (2009) considerando fatores de: produtividade (presenteísmo e absenteísmo), estilo de vida (inatividade física, alimentação inadequada, estresse excessivo, tabagismo), morbidade referida (diabetes, hipertensão arterial e hipercolesterolemia) e clima organizacional (carga de trabalho e motivação intrínseca).

A análise de dados foi realizada em três etapas, a saber: (1) análise descritiva de todas as variáveis: para variáveis quantitativas foi calculado o valor da média aritmética e o desvio padrão; para as variáveis qualitativas, foi calculada a frequência absoluta e a frequência relativa; (2) análise bivariada: foi realizada por meio do teste qui-quadrado para heterogeneidade ou para tendência linear, com nível de significância de $\mathrm{p}<0,05$; (3) análise de regressão: foi utilizada a análise de regressão logística uni variada para estimar os valores de odds ratio (risco relativo) (OR) e os respectivos intervalos de confiança.

\section{Resultados}

A análise descritiva mostrou que, no grupo pesquisado, há predomínio do sexo masculino (78,7\%), casados $(68,5 \%)$ e de escolaridade fundamental (incompleto 35,6\% e completo $14,4 \%)$. A idade média foi de 35,3 \pm 8,9 anos, sendo a idade mínima de 18 anos e máxima de 62 anos entre os trabalhadores que responderam à pesquisa (Tabela 1). 


\begin{tabular}{c|rr}
\multicolumn{3}{c}{ Tabela 1 - Perfil do grupo pesquisado } \\
\hline Dados & \multicolumn{1}{|c}{ n } & \multicolumn{1}{c}{$\%$} \\
\hline Sexo masculino & 333 & 78,7 \\
Sexo Feminino & 90 & 21,3 \\
\hline Casado & 290 & 68,5 \\
Solteiro & 117 & 27,7 \\
Divorciado & 16 & 3,8 \\
Viúvo & - & - \\
\hline $18-29$ anos & 114 & 27,0 \\
30-39 anos & 182 & 43,0 \\
40-49 anos & 89 & 21,0 \\
$\geq 50$ anos & 38 & 9,0 \\
\hline Fundamental incompleto & 150 & 35,6 \\
Fundamental completo & 61 & 14,4 \\
Ensino Médio incompleto & 50 & 11,8 \\
Ensino Médio completo & 116 & 27,4 \\
Superior incompleto & 25 & 5,9 \\
Superior completo & 17 & 4,0 \\
Pós Graduação & 4 & 0,9 \\
\hline \multicolumn{2}{c}{ Fonte: Autoria própra } \\
\hline (2010)
\end{tabular}

Com relação ao estilo de vida, constatou-se que $65,0 \%$ dos trabalhadores realizam mais que 150 minutos de atividade física por semana, considerando-se as atividades caminhada, moderada e vigorosa, de acordo com os critérios do IPAQ. Ao levar em conta somente as variáveis de atividade moderada e vigorosa, 46,3\% realizam mais que 150 minutos de atividade física por semana.

Quando questionados sobre o nível de estresse na vida, 11,8\% dos trabalhadores relataram estar nessa condição sempre ou quase sempre.

Um número relativamente baixo de trabalhadores é fumante $(8,3 \%)$ (Tabela 2$)$.

Tabela 2 - Indicadores de estilo de vida

\begin{tabular}{c|rr}
\hline Variável & \multicolumn{1}{|c}{ n } & \multicolumn{1}{c}{$\%$} \\
\hline Inativo no lazer & 148 & 35,0 \\
Estresse elevado & 50 & 11,8 \\
Fumantes & 35 & 8,3 \\
Excesso de peso & 167 & 39,5 \\
\hline
\end{tabular}

Fonte: Autoria própria (2010).

Solicitou-se que o trabalhador informasse o peso e a estatura. Após o cálculo do índice de massa corpórea (IMC), constatou-se que 41,2\% possuem excesso de peso e 9,7\% são obesos. Estes dados podem estar relacionados com alguns hábitos alimentares como o consumo da carne vermelha com excesso de gordura $(42,1 \%)$ e a baixa ingestão de frutas (apenas $11,2 \%$ comem frutas cinco ou mais dias da semana) e verduras e legumes (38,0\% têm este hábito cinco ou mais dias da semana). Questionados sobre diagnóstico prévio de doenças ou fatores de risco, 13,7\% relataram ser hipertensos, $1,9 \%$ diabéticos e $8,7 \%$ ter colesterol elevado (Tabela 3, 4 e 5).

Tabela 3 - Distribuição por peso corporal (IMC)

\begin{tabular}{c|rr}
\hline Peso Corporal (IMC) & \multicolumn{1}{|c}{ n } & \multicolumn{1}{c}{$\%$} \\
\hline Abaixo do peso & 7 & 2,1 \\
Peso normal & 154 & 47,0 \\
Excesso de peso & 135 & 41,2 \\
Obesidade & 32 & 9,7 \\
\hline
\end{tabular}

Fonte: Autoria própria (2010). 
Tabela 4 - Distribuição por hábitos alimentares

\begin{tabular}{c|cc}
\hline Fator & n & \% \\
\hline Consumo de verduras e legumes $(<5 \mathrm{x} / \mathrm{sem})$ & 241 & 57,0 \\
Consumo de frutas $(<5 \mathrm{x} / \mathrm{sem})$ & 273 & 64,5 \\
Consumo de carne vermelha com gordura visível & 178 & 42,1 \\
\hline
\end{tabular}

Fonte: Autoria própria (2010).

Tabela 5 - Morbidade referida

\begin{tabular}{c|rr}
\hline Variável & n & \% \\
\hline Hipertensão referida & 58 & 13,7 \\
Diabete referido & 8 & 1,9 \\
Colesterol alto referido & 37 & 8,7 \\
\hline \multicolumn{2}{c}{ Fonte: Autoria própria (2010). }
\end{tabular}

Os trabalhadores responderam se compareceram ao trabalho apesar de se sentir doentes. Constatou-se que 33,3\% dos trabalhadores relataram esta condição duas ou mais vezes nos últimos seis meses, com predomínio do sexo feminino (52,2\%). A avaliação do risco relativo demonstrou que as mulheres têm cerca de 2,8 vezes mais chance de presenteísmo em comparação com os homens. Questionados se faltaram ao trabalho por motivo de doença, nos últimos seis meses, cerca de $8 \%$ relataram ter se ausentado do trabalho por duas ou mais vezes (Tabela 6). Uma informação bastante relevante relacionada à produtividade está contida nas variáveis presenteísmo e absenteísmo.

Tabela 6 - Fatores relacionados à produtividade

\begin{tabular}{c|cc}
\hline Presenteísmo & n & $\mathbf{\%}$ \\
\hline Até uma vez & 282 & 66,7 \\
$\geq$ duas vezes & 141 & 33,3 \\
\hline Absenteísmo & n & $\%$ \\
\hline Até uma vez & 389 & 92,0 \\
$\geq$ duas vezes & 34 & 8,0 \\
\hline Fonte: Autoria própria (2010).
\end{tabular}

Considerando os fatores relacionados à produtividade, 63,2\% dos trabalhadores apresentaram três ou mais fatores de risco (Tabela 7).

Tabela 7 - Distribuição por número de fatores de risco

\begin{tabular}{c|rr}
\hline Número de Fatores & \multicolumn{1}{|c}{ N } & \multicolumn{1}{c}{$\%$} \\
\hline Nenhum fator & 17 & 4,0 \\
Um fator & 45 & 10,6 \\
Dois fatores & 94 & 22,2 \\
Três fatores & 130 & 30,7 \\
Quatro fatores & 76 & 18,0 \\
Cinco fatores & 49 & 11,6 \\
Seis fatores & 7 & 1,8 \\
Sete fatores & 4 & 0,9 \\
Nove fatores & 1 & 0,2 \\
\hline
\end{tabular}

Fonte: Autoria própria (2010).

As questões organizacionais foram analisadas com a utilização de dois domínios (motivação intrínseca e carga de trabalho) do questionário QVP-35. Com relação ao domínio carga de trabalho, em uma escala de Likert (zero a dez), constatou-se média igual ou superior a seis nas facetas - 
quantidade de trabalho, rapidez e afobação e pressão para manter a qualidade do trabalho, demonstrando uma percepção de carga de trabalho excessiva (Tabela 8).

Tabela 8 - Domínio carga de trabalho

\begin{tabular}{c|cc}
\hline Variáveis & $\mathrm{n}$ & $\%$ \\
\hline Quantidade de Trabalho & & \\
Carga não elevada & 104 & 24,6 \\
$\quad$ Carga elevada & 319 & 75,4 \\
$\quad$ Estresse & & \\
Carga não elevada & 243 & 57,4 \\
$\quad$ Carga elevada & 180 & 42,6 \\
$\quad$ Rapidez & & \\
Carga não elevada & 177 & 41,8 \\
$\quad$ Carga elevada & 246 & 58,2 \\
Pressão para realizar o trabalho & & \\
Carga não elevada & 234 & 55,3 \\
$\quad$ Carga elevada & 189 & 44,7 \\
Pressão para manter a qualidade do trabalho & & \\
Carga não elevada & 115 & 27,2 \\
Carga elevada & 308 & 72,8 \\
\hline
\end{tabular}

Fonte: Autoria própria (2010).

Com relação ao domínio motivação intrínseca todas as facetas mostraram média igual ou superior a seis - vontade de ser criativo, orgulho do trabalho, vontade de melhorar e satisfação no trabalho (Tabela 9).

\begin{tabular}{c|rc} 
Tabela 9 - Domínio motivação intrínseca \\
\hline Variáveis & \multicolumn{1}{c}{$\mathrm{n}$} & $\%$ \\
\hline Vontade de ser criativo & & \\
Baixa & 59 & 13,9 \\
Adequada & 364 & 86,1 \\
Orgulho do trabalho & & \\
Baixo & 67 & 15,8 \\
Adequada & 356 & 84,2 \\
Motivação & & \\
Baixa & 66 & 15,6 \\
Adequada & 357 & 84,4 \\
Satisfação com o trabalho & & \\
Baixa & 81 & 19,1 \\
Adequada & 342 & 80,9 \\
\hline Fonte: Autoria própria (2010).
\end{tabular}

A análise bivariada comparando os valores de prevalência de presenteísmo e razão de chance segundo as categorias das variáveis independentes dos funcionários nos fatores de estilo de vida constatou significância estatística somente na variável estresse (quase sempre - OR - 5,97 e sempre - OR - 2,21). O risco de presenteísmo é maior entre as mulheres (OR-2,78). Constatou-se um aumento no risco, apesar de não ser estatisticamente significativo em pessoas com hipertensão arterial (OR - 1,63), diabetes (OR - 1,2) e dislipidemia (OR - 1,70). Na Tabela 10 são apresentadas as variáveis independentes e presenteísmo: 
Tabela 10 - Variáveis independentes e presenteísmo

\begin{tabular}{c|ccc}
\hline Variáveis Independentes & Presenteísmo N (\%) & OR IC 95\% & p \\
\hline Gênero & & & $0,001^{\mathrm{a}}$ \\
Homens & $94(28,2)$ & 1.00 & \\
Mulheres & $47(52,2)$ & $2.78(1.72-4.48)$ & $0.001^{\mathrm{a}}$ \\
Classificação do Nível de Estresse & $112(30,0)$ & 1.00 & 0.117 \\
Baixo Estresse & $29(58,0)$ & $3.22(1.76-5.89)$ & \\
Alto Estresse & $45(28,7)$ & 1.00 & 0.410 \\
Carga de trabalho & $96(36,1)$ & $1.41(0.92-2.15)$ & \\
Não elevada & $114(32,5)$ & 1.00 & $1.25(0.74-2.11)$ \\
Elevada & $27(37,5)$ & & \\
Motivação intrínseca & Adequada & & \\
Baixa & &
\end{tabular}

Fonte: Autoria própria (2010).

Há forte correlação entre o presenteísmo referido (risco relativo de 6,54 - intervalo de confiança - 2,96 a 14,93, p <0.001) e o risco de faltas ao trabalho (absenteísmo) reforçando a necessidade de uma abordagem sistemática dos fatores relacionados ao presenteísmo.

Com relação a absenteísmo e fatores relacionados ao estilo de vida e qualidade de vida profissional, a análise bivariada não constatou significância estatística (Tabela 11).

Tabela 11 - Variáveis independentes e absenteísmo

\begin{tabular}{c|ccc}
\hline Variáveis Independentes & Absenteísmo n (\%) & $\begin{array}{c}\text { OR IC } \\
\mathbf{9 5 \%}\end{array}$ & \\
\hline Gênero & $24(7,2)$ & 1,00 & 0,227 \\
Homens & $10(11,1)$ & $1,60(0,74-3,50)$ & \\
Mulheres & & 1,00 & 0,272 \\
Classificação do nível de estresse & $28(7,5)$ & $1,68(0,66-4,28)$ & \\
Baixo Estresse & $6(12)$ & 1,00 & 0,888 \\
Alto Estresse & & & \\
Carga de Trabalho & $13(8,3)$ & $1,95(0,46-1,95)$ & \\
Não Elevada & $21(7,9)$ & 1,00 & 0,564 \\
Elevada & $27(7,7)$ & $1,29(0,54-3,09)$ & \\
Motivação Intrínseca & $7(9,7)$ &
\end{tabular}

A análise bivariada comparando a inatividade física e os diferentes fatores constatou que os fumantes (OR - 2,71), hipertensos (OR - 1,49) e diabéticos (OR - 13,58) são menos ativos. Finalmente, constatou-se que há forte correlação estatística entre o número de fatores de risco em saúde estilo de vida e inatividade física (Tabelas 12 e 13).

Tabela 12 - Fatores de risco e inatividade física

\begin{tabular}{c|ccl}
\hline Fatores de Risco & $\mathbf{N}(\%)$ & OR & p \\
\hline Nenhum & - & - & $0,001^{\mathrm{b}}$ \\
Um a dois & $24(16,2)$ & 1,00 & \\
Três a quatro & $77(52,0)$ & $1,67(1.01-4.35)$ & \\
Cinco ou mais & $47(31,7)$ & $3,45(1.32-8.98)$ & \\
\hline
\end{tabular}

Fonte: Autoria própria (2010).

Tabela 13 - Idade e inatividade física

\begin{tabular}{c|ccc}
\hline Faixa Etária & $\mathbf{n}(\boldsymbol{\%})$ & OR & $\mathbf{p}$ \\
\hline $18-29$ anos & $50(43,9)$ & 1,00 & $0,005^{\mathrm{b}}$ \\
$30-39$ anos & $48(26,4)$ & $0,46(0.28-0.75)$ & \\
40-49 anos & $32(36,0)$ & $0,72(0.41-1.27)$ & \\
$\geq 50$ anos & $18(48,6)$ & $1,21(0.58-2.55)$ & \\
\hline
\end{tabular}

Fonte: Autoria própria (2010). 


\section{Discussão}

A presente pesquisa utilizou uma amostra de trabalhadores de uma indústria têxtil do Estado da Paraíba para analisar fatores de risco em saúde e estilo de vida que podem afetar a produtividade e possibilitar a elaboração de estratégias para promover a qualidade de vida do trabalhador da indústria.

O estudo observou a predominância de trabalhadores do sexo masculino, casados, com idade média de 35 anos, sendo que 70,3\% têm menos de 40 anos. Constatou-se que quase metade dos trabalhadores possui instrução de ensino fundamental (completo ou incompleto). O nível de escolaridade observado foi inferior ao constatado na pesquisa nacional de estilo de vida da indústria do SESI (2007).

Vários estudos (BERGSTROM et al., 2009; PRONK, 2009) relataram a importância de se estratificar os trabalhadores por número de fatores de risco. Constatou-se que a cada fator corresponde aumento no risco de doenças, de redução na produtividade e no incremento nos custos de assistência médica. Foram utilizados nove fatores de risco (excesso de peso, alimentação inadequada, estresse, inatividade física, diabete, hipertensão, colesterol elevado, consumo de excesso de gorduras, tabagismo).

Os indicadores de estilo de vida (atividade física, stress, alimentação, tabagismo e excesso de peso) estão em percentuais próximos ao observado na Pesquisa de Estilo de Vida do SESI (2007). A presente pesquisa revelou que $63,2 \%$ de trabalhadores apresentam três ou mais fatores de risco em saúde e estilo de vida, revelando risco considerável de aumento de doenças crônicas (infarto do miocárdio, derrame cerebral, diabete e câncer) nessa população, com eventual comprometimento da produtividade relacionada ao fator humano. $\mathrm{O}$ estudo expôs que, aproximadamente, um terço dos trabalhadores relataram ter duas ou mais situações em que foram trabalhar, apesar de se sentir doentes. A avaliação estatística aponta que, nesse grupo, o presenteísmo apresenta um risco 6,5 vezes maior de absenteísmo, ou seja, as pessoas que hoje comparecem ao trabalho, apesar de se sentirem doentes, tem grande chance de faltarem ao trabalho em ocasiões futuras.

A pesquisa utilizando domínios (carga de trabalho e motivação intrínseca) do questionário QVP-35 revelou que os trabalhadores mostram-se motivados com o trabalho, mas demonstram percepção de carga de trabalho excessiva (particularmente relacionada à quantidade de trabalho e pressão para manter a qualidade). Provavelmente, medidas gerenciais visando estimular uma maior variedade nas atividades ou pausas regulares podem colaborar para a melhoria desse indicador.

Neste contexto, a promoção do estilo de vida saudável, com especial ênfase para a atividade física constitui-se numa ferramenta poderosa para a melhoria dos indicadores analisados. A presente pesquisa revelou que as pessoas com maior idade e com mais fatores de risco têm risco aumentado pela inatividade física.

Vários autores demonstraram o impacto empresarial das ações em promoção de saúde e qualidade de vida:

- 25\% dos gastos com saúde estão relacionados com dez fatores de risco modificáveis. (GOETZEL et al., 2009);

- Redução média de 56 dólares nos custos de assistência médica para cada melhora no índice de bem-estar (YEN et al., 2003);

- Aumento de 76 dólares em uso de medicamentos para cada fator de risco adicional (BURTON et al., 2006);

- Absenteísmo variou de 0 a 6,3\%, respectivamente para pessoas com zero a oito fatores de risco (BOLES; PELLETIER; LYNCH, 2004);

- Presenteísmo variou de 1,5 a 25,9\% respectivamente para pessoas com zero a oito fatores de risco. (BOLES; PELLETIER; LYNCH, 2004); 
- Absenteísmo provocou 4,1 dias perdidos para pessoas com zero fator de risco chegando a 12,6 dias perdidos para pessoas com quatro ou mais fatores de risco (TSAI et al., 2005);

- Perda de produtividade variando de $11,9 \%$ a $28,3 \%$ para pessoas com zero a sete fatores de risco. Empregados de médio risco (Três-quatro fatores) são 6,2\% menos produtivos que os de baixo risco (Zero-dois). Os de alto risco (cinco ou mais fatores de risco) são 12,2\% menos produtivos que os de médio risco (3-4 fatores). (BURTON et al., 2006);

- Melhora de 9\% no presenteísmo para cada redução no número de fatores de risco (PELLETIER; BOLES; LYNCH 2004).

\section{Referências}

BERGSTROM, G. et al. Sickness presenteeism today, sickness absenteeism tomorrow? A Prospective study on sickness presenteeism and future sickness absenteeism. Journal of Occupational and Environmental Medicine, v. 51, p. 629-638, 2009.

BOLES, M.; PELLETIER, B.; LYNCH, W. The relationship between health risks and work productivity. Journal of Occupational and Environmental Medicine, v. 46, p. 737-745, 2004.

BURTON, W. N.; CHEN, C. Y.; CONTI, D. J.; SCHUlTZ, A. B.; EDINGTON, D. W. The association between health risk change and presenteeism change. Journal of Occupational and Environmental Medicine, v. 48, p. 252-263, 2006.

CABEZAS-PEÑA, C. Sindrome de desgaste professional, estrés laboral y calidad professional. Formación Médica Continuada en Atención Primaria, v. 5, n. 8, p. 491,492, 1999.

GOETZEL, R. et al. The relationship between modifiable health risk factors and medical expenditures, absenteeism, short term disability and presenteeism among employees at Novartis. Journal of Occupational and Environmental Medicine, v. 51, n. 4, p. 487-499, 2009.

GUIMARAES, L. A. M. et al. Atualizações em qualidade de vida e trabalho. In: GUIMARÃES, L. A. M.; GRUBITS, S. (Org.). São Paulo: Casa do Psicólogo, 2004.

MATSUDO, S. et al. Questionário Internacional de Atividade Física (IPAQ) - Estudo de validade e reprodutibilidade no Brasil. Revista Brasileira de Atividade Física e Saúde, v. 6, n. 2, 2001.

MINISTÉRIO DA SAÚDE. Vigitel Brasil 2009: vigilância de fatores de risco das indústrias de 23 estados e do Distrito Federal. Brasília: Serviço Social da Indústria, 2010.

NAHAS, M. V. et al. Estilo de vida e hábitos de lazer dos trabalhadores das indústrias de 23 estados brasileiros e do Distrito Federal: relatório geral. Brasília, Serviço Social da Indústria, 2008.

PELLETIER, B.; BOLES, M.; LYNCH, W. Change in health risks and work productivity over time. Journal of Occupational and Environmental Medicine, v. 46, p. 746-754, 2004.

PRONK, N. P. ACMS's Worksite Health Handbook. A Guide to Building Healthy and Productive Companies - Human Kinetics, 2009.

SCHULTZ, A.; CHEN, C.; EDINGTON, D. The cost and impact of health conditions on presenteeism to employers. Pharmacoeconomics, n. 27, n. 5, p. 365-378, 2009. 
SESI. SERVIÇO SOCIAL DA INDÚSTRIA. Diretrizes técnicas e de gestão SESI lazer ativo. O programa: metodologia de atendimento às empresas. Brasília: SESI/DN, 2007.

TSAI, S. P. et al. Illness absence patterns among employees in a petrochemical facility: impact of selected health risk factors. Journal of Occupational and Environmental Medicine, v. 47, p. 838846, 2005.

YEN, L. et al. Association between wellness score from a health risk appraisal and prospective medical claims costs. Journal of Occupational and Environmental Medicine, v. 45, n. 10, p. 1049-1057, 2003. 